\title{
Micro-to-nano Scale Strain Characterization of 2024 Aluminum-alloys with Incoherent/Coherent Precipitates
}

Florent Ravaux ${ }^{1}$, Dalaver Anjum ${ }^{1}$, Cyril Aubry ${ }^{1}$, Gregory Haidemenopoulos ${ }^{2}$, Helen Kamoutsi ${ }^{2}$, H. Mavros $^{1}$, Nirpendra Singh ${ }^{1}$, Issam Qattan ${ }^{1}$, Gobind Das ${ }^{1}$ and Shashikant Patole ${ }^{1}$

${ }^{1}$ Khalifa University, Abu Dhabi, Abu Dhabi, United Arab Emirates, ${ }^{2}$ University of Thessaly, Volos, Magnisia, Greece

This study is focused on the crystal structure of 2024 aluminum-alloy (Al-alloy) with copper $(\mathrm{Cu})$ as primary alloying element. These alloys have excellent properties for corrosion and aerospace applications. The main drawback of this alloy is its fast corrosion rate. There is a correlation between the strain and the hydrogen trapping mechanism that is responsible for corrosion in 2024 alloys. Therefore, an Al-alloy with low density of defects is desirable at the vicinity of the precipitates to reduce corrosion. Many techniques such as X-ray diffraction, nano-indentation or Raman spectroscopy are used to determine strain on bulk material. However, information gathered using these techniques are not sufficient to understand defect induced corrosion mechanism. In this study, we propose a methodology to correlate strain to defect localization induced by $\mathrm{Cu}$ precipitates by combining different Transmission Electron Microscopy (TEM) techniques. To develop this technique, we used an Al-alloy which belongs to the 2024 category: Al-4.35 $\mathrm{Cu}-1.5 \mathrm{Mg}-0.64 \mathrm{Mn}-0.5 \mathrm{Si}-0.5 \mathrm{Fe}$ (wt.\%). The sample was treated at $495 \mathrm{C}$ followed by water quenching. The aging process was carried out but at $210 \mathrm{C}$ for an hour to accelerate the precipitate formation. Specimens for TEM analysis were prepared using a standard focused ion beam (FIB) lamella methodology and the TEM characterization was performed using aberration-corrected microscope of model Titan 80300 ST from Thermo-Fisher Scientific. TEM characterization was performed on specimens to establish the degree of coherency of S" precipitates in Al matrix. This is accomplished by determining strain fields around the precipitates. Our results suggest that the presence of strain fields due to dislocations is indicative of incoherent or semicoherent precipitates. Whereas, no dislocation strain fields in the maps implies a fully coherent precipitate in Al matrix. To achieve this goal, several TEM techniques were used as follows:

- Geometric Phase Analysis (GPA) is used to determine the strain around the precipitates and to localized possible dislocations and evaluate their impacts on strain gradient; and

- Nano beam diffraction (NBD) is used to determine strain at the microscale due to its higher field of view which allows to map strains at the grain size scales.

In this study, we characterized a region with three different precipitates oriented in the plan normal to the sample. We first acquired high-resolution scanning TEM images of these three precipitates and used geometrical phase analysis (GPA) algorithm to obtain strain mapping close to one nanometer scale [1]. Due to the configuration of the TEM used for this experiment (absence of probe corrector), the field of view with this technique is limited to $20 \mathrm{~nm}$ x $20 \mathrm{~nm}$ area. Figures 1(a-c) depict the strain gradients of the three precipitates in both directions, i.e. longitudinal $\left(\varepsilon_{\mathrm{xx}}\right)$ and vertical $\left(\varepsilon_{\mathrm{yy}}\right)$. GPA provided strain mapping at the nanometer scale which made it possible to detect dislocation sites [2]. Large strain variations are observed inside all precipitates which are due to the incoherency between the precipitates and the matrix. Moreover, strain variations are also observed in the Al-matrix and are identified as dislocations (cf. dashed black circles). For a better understanding of the influence of precipitates on strain at a larger scale, we performed the NBD mapping on the same region covering the 3 precipitates. The surface area analysis was performed for around $60 \mathrm{~nm}$ x $60 \mathrm{~nm}$ with a pixel size of $2 \mathrm{~nm}$. Figure 2(a) shows the STEM image of the region containing the precipitates. Figure 2(b) depicts the same region imaged with NBD technique 
with a lower spatial resolution. Figure 2(c) and 2(d) show the mechanical deformation maps of NBD measurement of the red square processed using epsilon software [3, 4]. The different precipitates and dislocations were carefully localized on the new mapping. Large strain variations were observed at the vicinity of the precipitates. Indeed, the strain variations was found varying from tensile (positive values) to compressive (negative values) when observing deformation from left to right. Moreover, the dislocations observed in Figure 1 seem to be located in the regions where similar strain inversions were observed as well. As expected, this study shows that $\mathrm{Cu}$ precipitates can be a source of large strain relaxation in $\mathrm{Al}$ alloys. Crystal dislocations were recorded inside the precipitate itself and at its vicinity where strain inversions can also be observed. Further investigation using the same methodology will be carried out on similar samples with coherent precipitates.

\section{Acknowledgements:}

The work related to TEM-specimen preparation and subsequent TEM analysis was funded by ADEK, Abu Dhabi through the Project No. 843400341.

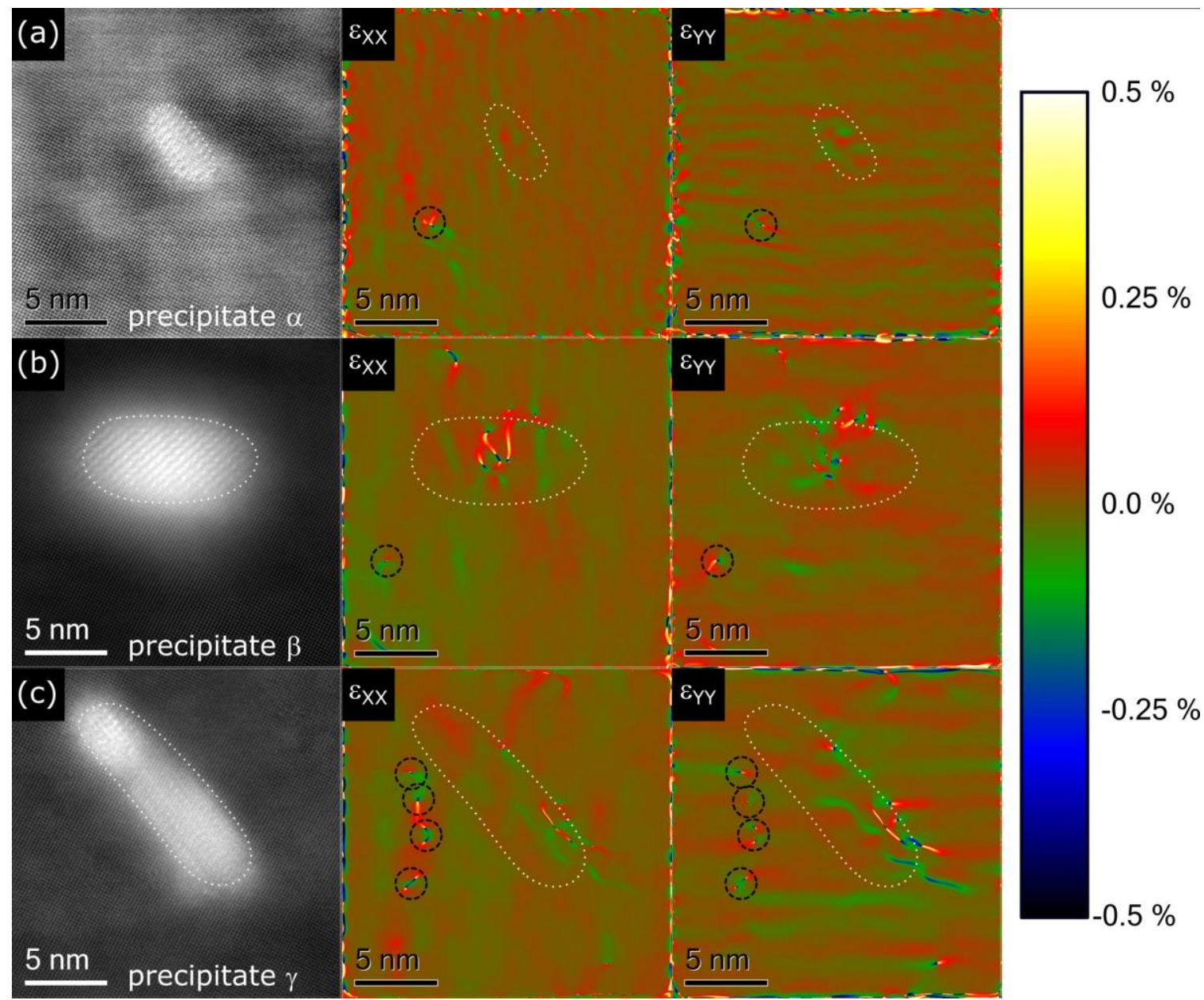

Figure 1. (a-c) HR-STEM images of 3 different precipitates labelled $(\alpha),(\beta)$, and $(\gamma)$. The associated strain mappings are calculated using GPA. The inserted dashed white circles show the location of the precipitates, and the dashed black circles show the location of dislocations in the Al matrix. 


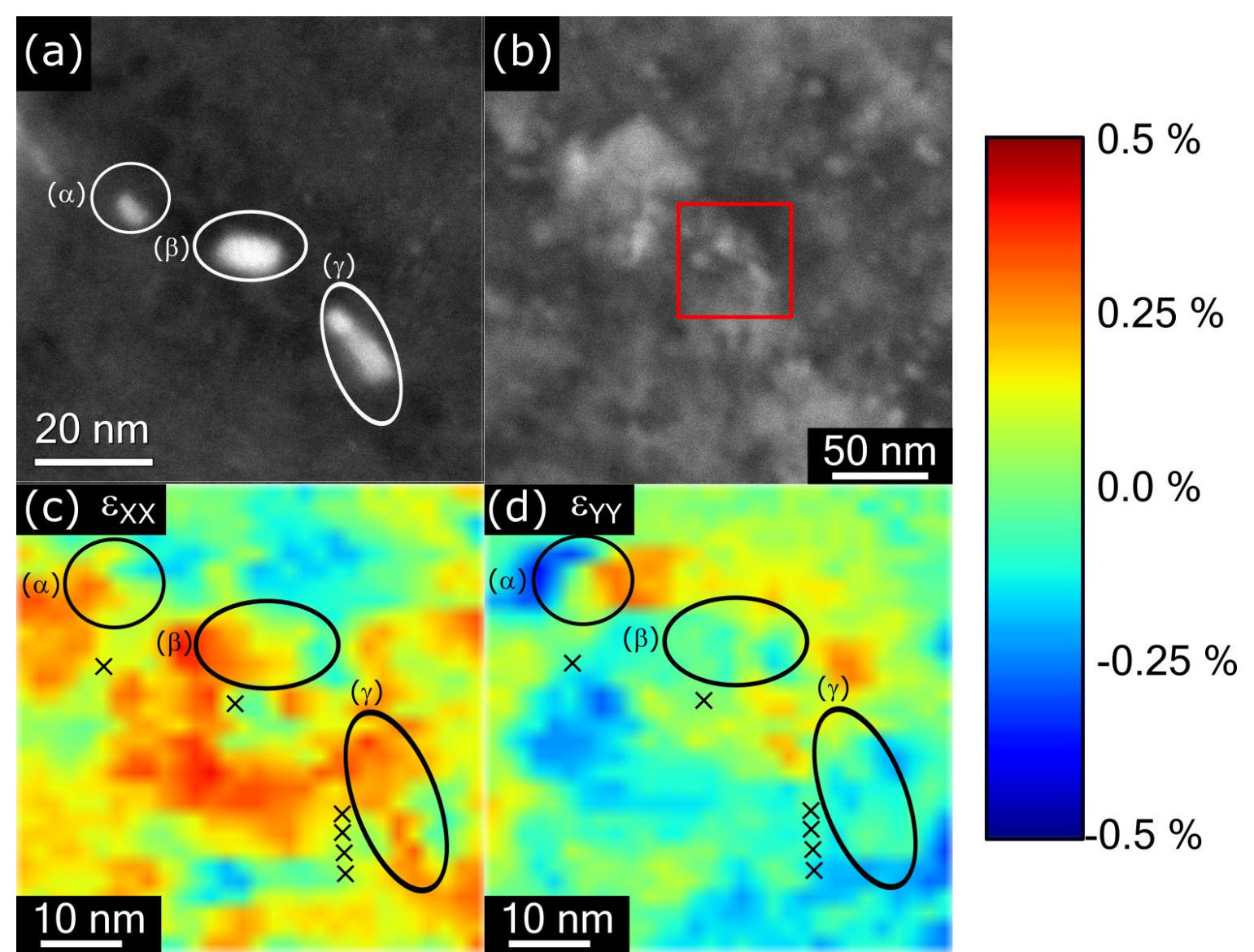

Figure 2. (a) STEM image showing the precipitate (a), (b) and (g). (b) NBD image showing the scanned area (red square) for strain analyzes. (c) Longitudinal and (d) vertical strain mappings. Inserts show the locations of the precipitates and the dislocations identified with GPA.

\section{References}

[1] V. B.Ozdol, D. Tyutyunnikov, C.T.Koch, and P. A. van Aken, "Strain mapping for advanced CMOS technologies" Cryst. Res. Technol.49 (1), (2014) pp. 38-42 doi.org/10.1002/crat.201300226

[2] M. Hytch, F. Snoeck, R. Kilaas, "Quantitative measurement of displacement and strain fields from HREM micrographs”, Ultramicroscopy 74 (1998), pp. 131-146 doi.org/10.1016/S0304-3991(98)00035-7 [3] J.-L. Rouviere, B. Haas, E. Robin, D. Cooper, N. Bernier and M. Williamson, "The Measurement of Strain, Chemistry and Electric Fields by STEM based Techniques”, Microsc. Microanal. 23 (S1), (2017). doi.org/10.1017/S1431927617007735

[4] M. J. Williamson, P. van Dooren, and J. Flanagan, "Quantitative analysis of the accuracy and sensitivity of strain measurements from nanobeam electron diffraction”, 2015 IEEE 22nd International ymposium on the Physical and Failure Analysis of Integrated Circuits, (2015), pp.197-200 doi.org/10.1109/IPFA.2015.7224366 